\title{
Histones, histone chaperones and nucleosome assembly
}

\author{
Rebecca J. Burgess, Zhiguo Zhang ${ }^{\bowtie}$ \\ Department of Biochemistry and Molecular Biology, Mayo Clinic College of Medicine, 200 First Street SW, Rochester, MN 55905, \\ USA \\ \ Correspondence: zhang.zhiguo@mayo.edu
}

\begin{abstract}
Chromatin structure governs a number of cellular processes including DNA replication, transcription, and DNA repair. During DNA replication, chromatin structure including the basic repeating unit of chromatin, the nucleosome, is temporarily disrupted, and then reformed immediately after the passage of the replication fork. This coordinated process of nucleosome assembly during DNA replication is termed replication-coupled nucleosome assembly. Disruption of this process can lead to genome instability, a hallmark of cancer cells. Therefore, addressing how replication-coupled nucleosome assembly is regulated has been of great interest. Here, we review the current status of this growing field of interest, highlighting recent advances in understanding the regulation of this important process by the dynamic interplay of histone chaperones and histone modifications.
\end{abstract}

\section{CHROMATIN STRUCTURE AND NUCLEOSOME ASSEMBLY}

In eukaryotic cells, genomic DNA is packaged into chromatin, an organized complex of DNA and proteins (Luger et al., 1997; Kornberg and Lorch, 1999; Wu and Grunstein, 2000; Chodaparambil et al., 2006). The fundamental unit of chromatin is the nucleosome, consisting of 146 base pairs of DNA wrapped around a histone octamer containing a tetramer of histones $\mathrm{H} 3-\mathrm{H} 4$ and two dimers of histones $\mathrm{H} 2 \mathrm{~A}$ H2B. During $S$ phase of the cell cycle, chromatin structure must be propagated to daughter cells to maintain gene expression state and genome integrity. How chromatin structure is inherited during $S$ phase of the cell cycle remains largely unknown (Goldberg et al., 2007). During DNA replication, eukaryotic cells disassemble nucleosomes to facilitate progression of the DNA replication machinery (Falbo and Shen, 2006). Following replication, newly-synthesized histones, as well as parental histones, must be deposited onto the nascent DNA strands to mediate nucleosome formation and reassembly of chromatin higher order structure. This coupling of DNA replication and nucleosome assembly is termed replication-coupled (RC) nucleosome assembly. It is believed that DNA RC nucleosome assembly plays an important role in the inheritance of chromatin structure.

During RC nucleosome assembly, parental $\mathrm{H} 3-\mathrm{H} 4$, as well as newly-synthesized $\mathrm{H} 3-\mathrm{H} 4$, are deposited first followed by rapid deposition of $\mathrm{H} 2 \mathrm{~A}-\mathrm{H} 2 \mathrm{~B}$ dimers to form nucleosomes. Once assembled into nucleosomes, $\mathrm{H} 3-\mathrm{H} 4$ molecules remain stably bound to DNA, whereas nucleosomal $\mathrm{H} 2 \mathrm{~A}$ and $\mathrm{H} 2 \mathrm{~B}$ can exchange with free $\mathrm{H} 2 \mathrm{~A}$ and $\mathrm{H} 2 \mathrm{~B}$ (Rocha and Verreault, 2008; Ransom et al., 2010). Therefore, it is important to understand how $\mathrm{H} 3-\mathrm{H} 4$ are deposited, the factors involved in deposition and the regulatory mechanisms contributing toward nucleosome assembly. Because the deposition of newly-synthesized $\mathrm{H} 3-\mathrm{H} 4$ molecules and the regulation of their deposition are relatively well understood compared to the transfer of parental histones $\mathrm{H} 3-\mathrm{H} 4$ behind replication forks, this perspective will focus on how newly-synthesized $\mathrm{H} 3-\mathrm{H} 4$ are deposited.

\section{THE FUNCTION OF THREE HISTONE CHAPERONES IN RC NUCLEOSOME ASSEMBLY IN YEAST}

Deposition of newly-synthesized $\mathrm{H} 3-\mathrm{H} 4$ during DNA replication requires histone chaperones. Histone chaperones are a group of proteins that help regulate nucleosome deposition by binding to the positively charged histones and shielding their charge from the highly negatively charged DNA (Tyler, 2002). 
The classic histone chaperone involved in $\mathrm{RC}$ nucleosome assembly is chromatin assembly factor 1 (CAF-1). CAF-1 consists of three subunits (Kaufman et al., 1997) and was first identified in human cells as a factor that promotes nucleosome assembly during DNA replication (Stillman, 1986). CAF-1 binds $\mathrm{H} 3-\mathrm{H} 4$ and facilitates nucleosome assembly following DNA replication and DNA repair by interacting with PCNA, a component of the DNA replication machinery (Shibahara and Stillman, 1999). Yeast cells lacking CAF-1 exhibit reduced silencing at telomeres, as well as the silent mating type loci, and are sensitive to DNA damaging agents (Kaufman et al., 1997; Linger and Tyler, 2005), phenotypes shared among nucleosome assembly factor mutants.

The ability of CAF- 1 to deposit $\mathrm{H} 3-\mathrm{H} 4$ onto replicated DNA is assisted by Asf1, another $\mathrm{H} 3-\mathrm{H} 4$ histone chaperone (Tyler et al., 1999; Mello et al., 2002). Asf1 was first identified in a genetic screen based on its ability to disrupt transcriptional silencing in budding yeast when overexpressed (Le et al., 1997). Later, it was shown that Asf1 was a histone chaperone functioning in both replication-coupled and replicationindependent nucleosome assembly (Tyler et al., 1999). Structural studies have revealed that Asf1 binds $\mathrm{H} 3-\mathrm{H} 4$ dimers through an $\mathrm{H} 3$ interface that is involved in the formation of $(\mathrm{H} 3-\mathrm{H} 4)_{2}$ tetramers (English et al., 2006). In human cells, it is proposed that Asf1 can disrupt parental nucleosomes (Groth et al., 2007). Furthermore, Asf1 is required for acetylation of histone $\mathrm{H} 3$ lysine 56 (H3K56Ac), a mark of newly-synthesized histones that has been found to be important for DNA replication and DNA repair (Recht et al., 2006; Chen et al., 2008; Li et al., 2008).

A more recently described $\mathrm{H} 3-\mathrm{H} 4$ histone chaperone in yeast is Rtt106. Rtt106 was first identified in a genetic screen for regulators of Ty1 transposition in budding yeast (Scholes et al., 2001). In a separate genetic screen, we identified Rtt106 as a protein that functions in parallel to PCNA in transcriptional silencing (Huang et al., 2005). Furthermore, we have shown that Rtt106 is a histone $\mathrm{H} 3-\mathrm{H} 4$ chaperone involved in RC nucleosome assembly (Li et al., 2008). Like mutations in genes encoding other histone chaperones, the rtt106 $\Delta$ mutant cells exhibit significant loss of silencing at telomeres and at the silent mating type locus and are sensitive to DNA damage agents when combined with deletion of CAC1, the large subunit of CAF-1 (Huang et al., 2007; Li et al., 2008). Rtt106 is also proposed to have a role in replication-independent nucleosome assembly as well as the regulation of histone gene expression (Imbeault et al., 2008; Fillingham et al., 2009).

These remarkable discoveries have led to the following question: how are $(\mathrm{H} 3-\mathrm{H} 4)_{2}$ tetramers, one of the building blocks of nucleosomes, formed during $S$ phase of the cell cycle if Asf1 binds the same $\mathrm{H} 3$ surface involved in $(\mathrm{H} 3-\mathrm{H} 4)_{2}$ tetramer formation? We and others have made observations that support a model in which $\mathrm{H} 3-\mathrm{H} 4$ dimers are transferred from Asf1-H3-H4 to CAF-1 and Rtt106, which in turn deposit $\mathrm{H} 3-\mathrm{H} 4$ onto replicating DNA for nucleosome formation. First, the association of histone $\mathrm{H} 3-\mathrm{H} 4$ with CAF-1 and Rtt106 is reduced in asf1 $\Delta$ cells (Li et al., 2008). Second, Asf1 has been shown to interact directly with CAF-1 (Tyler et al., 2001; Mello et al., 2002). Together, these three histone chaperones, CAF1, Rtt106 and Asf1, coordinate the deposition of newlysynthesized $\mathrm{H} 3-\mathrm{H} 4$ during DNA replication, but how exactly these chaperones cooperate is unclear (Fig. 1).

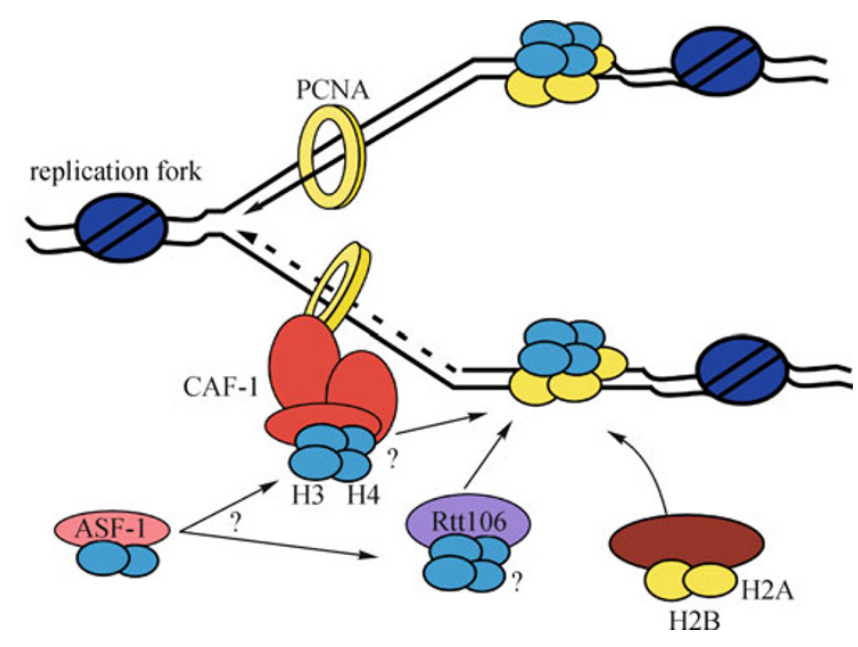

Figure 1. Coordination of histone chaperones for replication-dependent nucleosome assembly. During DNA replication, nucleosomes are disassembled and then reassembled behind the replication fork. The three histone chaperones, CAF-1, Asf1 and Rtt106 coordinate to deposit newly-synthesized $\mathrm{H} 3-\mathrm{H} 4$ onto the replicated DNA. It is still unclear how the Asf1-H3-H4 complex interacts with CAF-1 and Rtt106 for histone deposition. It is also unknown whether CAF-1 and Rtt106 bind H3-H4 dimers or tetramers. 


\section{MULTIPLE ACETYLATION EVENTS REGULATE ASSEMBLY OF NEWLY-SYNTHESIZED HISTONES H3 AND H4 IN BUDDING YEAST}

Modifications on histones regulate distinct cellular processes, including gene transcription, DNA replication, and DNA repair. These diverse functions are carried out by distinct modifications, including acetylation, phosphorylation, methylation and ubiquitination, which modulate chromatin structure and/or recruit proteins to chromatin to mediate a specific process (Strahl and Allis, 2000; Turner, 2000; Jenuwein and Allis, 2001; Vidanes et al., 2005). Histone acetylation is catalyzed by histone acetyltransferases (HATs), a group of enzymes that transfer the acetyl group from the cofactor acetyl-Coenzyme A (acetyl-CoA) to the $\varepsilon$-amino group of lysine residues on histones (Marmorstein and Roth, 2001; Roth et al., 2001; Carrozza et al., 2003; Marmorstein, 2004). It was discovered years ago that newly-synthesized histones were acetylated and rapidly deacetylated following deposition (Ruiz-Carrillo et al., 1975; Jackson et al., 1976). It is only recently that we have begun to appreciate the function of acetylated newly-synthesized $\mathrm{H} 3-\mathrm{H} 4$.

The most highly-conserved mark of newly-synthesized histones is acetylation of histone $\mathrm{H} 4$ lysines 5 and 12 ( $\mathrm{H} 4 \mathrm{~K} 5$, 12Ac). While acetylation on newly-synthesized histone $\mathrm{H} 3$ is conserved, the distinct acetylated sites are not. In HeLa cells, acetylation at the $\mathrm{H} 3-\mathrm{N}$ terminus of new $\mathrm{H} 3$ is barely detectable (Sobel et al., 1995). Moreover, acetylation of H3 lysine 56, a well-established and abundant mark of newlysynthesized H3 in budding yeast (Masumoto et al., 2005), is less abundant in mammalian cells (Jasencakova et al., 2010). Acetylation of distinct sites of newly-synthesized H3-H4 is catalyzed by different histone acetyltransferases. For instance, Hat 1 acetylates lysine 5 and 12 of $\mathrm{H} 4$ (Ai and Parthun, 2004). Rtt109 acetylates lysine 56 of H3 in yeast (Driscoll et al., 2007; Han et al., 2007), and in mammalian cells, this modification is catalyzed by CBP/p300 and/or Gcn5 (Das et al., 2009; Tjeertes et al., 2009). Recently, we have shown that new $\mathrm{H} 3$ acetylation at the $\mathrm{N}$-terminal tail is carried out by the lysine acetyltransferases Gcn5, Rtt109 and possibly Elp3 (Li et al., 2009; Burgess et al., 2010). Thus, acetylation of multiple sites at newly-synthesized $\mathrm{H} 3-\mathrm{H} 4$ is catalyzed by distinct lysine acetyltransferases (Fig. 2). How acetylation of these sites functions to promote nucleosome assembly is an interesting question.

Genetic studies suggest that acetylation of newlysynthesized $\mathrm{H} 3-\mathrm{H} 4$ is important for nucleosome assembly (Ma et al., 1998). Only recently have studies revealed that histone acetylation serves an important regulatory function during nucleosome assembly. For instance, In yeast cells, H3K56Ac, which peaks during $S$ phase of the cell cycle (Masumoto et al., 2005; Han et al., 2007; Chen et al., 2008), increases the binding affinity between $\mathrm{H} 3-\mathrm{H} 4$ with $\mathrm{CAF}-1$ and Rtt106 and promotes efficient deposition of $\mathrm{H} 3-\mathrm{H} 4$ onto

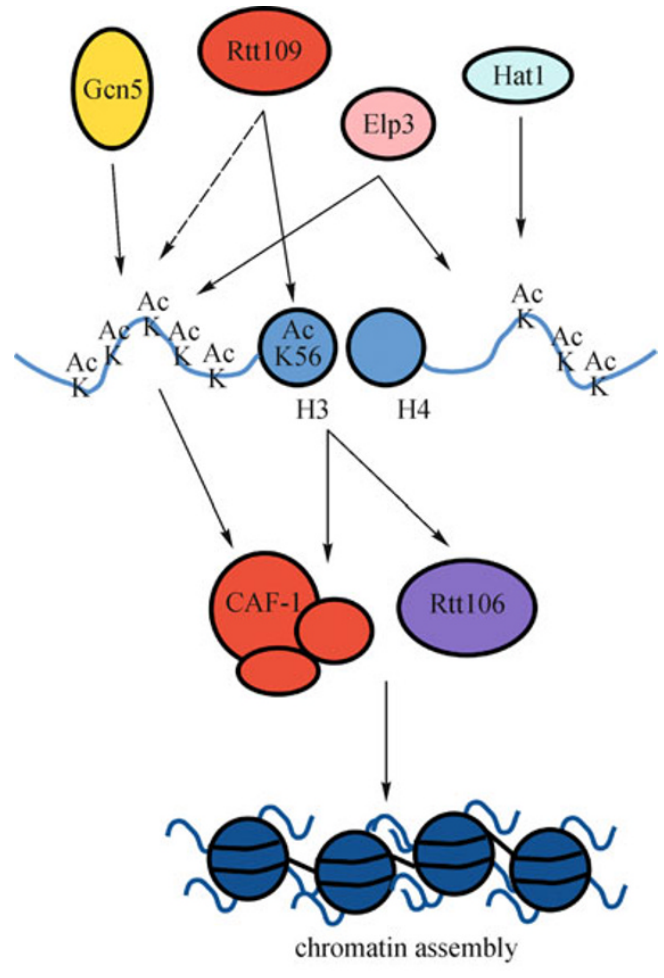

Figure 2. Histone acetylation of $\mathrm{H} 3-\mathrm{H} 4$ regulates replicationdependent nucleosome assembly by promoting the interaction of histones with histone chaperones. The histone acetyltransferases Gcn5, Rtt109, Elp3, and Hat1 are involved in marking newly-synthesized histones $\mathrm{H} 3-\mathrm{H} 4$. Some of these acetylation marks are known to promote histone interactions with histone chaperones for chromatin assembly. Acetylation of the $\mathrm{N}$ terminus of $\mathrm{H} 3$ is primarily carried out by Gcn5. Elp3 and Rtt109 also contribute to the acetylation of the H3 N terminus, but how these three distinct lysine acetyltransferases coordinate these acetylation events is not known. In addition, Rtt109 acetylates H3K56 on the core of histone H3. Hat1 and Elp3 acetylate lysine 5, 8 and 12 on $\mathrm{H} 4$; however, the functions of these $\mathrm{H} 4$ marks are unclear.

replicating DNA by these two histone chaperones (Li et al., 2008). In contrast, acetylation of lysine residues at the H3 Nterminus regulates the binding of $\mathrm{H} 3-\mathrm{H} 4$ with $\mathrm{CAF}-1$, but not Rtt106 (Burgess et al., 2010). Therefore, acetylation of H3 lysine 56 and acetylation of lysine residues at the $\mathrm{H} 3 \mathrm{~N}$ terminus function to promote nucleosome assembly by enhancing histone binding with distinct histone chaperones.

Finally, despite the conservation of $\mathrm{H} 4 \mathrm{~K} 5,12 \mathrm{Ac}$ from yeast to humans, its function is still unknown. In mammalian cells, prior to deposition, the canonical $\mathrm{H} 3, \mathrm{H} 3.1$, is associated with $\mathrm{H} 4 \mathrm{~K} 5,12 \mathrm{Ac}$, which is distinct from the pattern observed on nucleosomal H3.1-H4 (Loyola et al., 2006). Histone H4 molecules in CAF-1-H3-H4 complexes are acetylated at lysine residues 5,8 and 12 in both yeast and human cells (Verreault et al., 1996; Zhou et al., 2006). Furthermore, the 
diacetylated $\mathrm{H} 4$ pattern is also found on $\mathrm{H} 3.1-\mathrm{H} 4$ dimers associated with Asf1 (Jasencakova et al., 2010). Therefore, it is tempting to speculate that $\mathrm{H} 4 \mathrm{~K} 5,12 \mathrm{Ac}$ also regulates the interaction between $\mathrm{H} 3-\mathrm{H} 4$ with histone chaperones. Alternatively, acetylation of these lysine residues may facilitate nuclear import of newly-synthesized $\mathrm{H} 3-\mathrm{H} 4$. In yeast, Hat1, the enzyme catalyzing $\mathrm{H} 4 \mathrm{~K} 5,12 \mathrm{Ac}$, associates with newlysynthesized $\mathrm{H} 3-\mathrm{H} 4$ within the cytoplasm and remains associated as the entire complex moves to the nucleus before transferring the associated histones to other histone chaperones (Ai and Parthun, 2004; Shahbazian and Grunstein, 2007). Future studies are needed to address these possibilities.

\section{PERSPECTIVE: THE FUNCTION OF MODIFICATIONS ON NEWLY-SYNTHESIZED HISTONES IN NUCLEOSOME ASSEMBLY IN MAMMALIAN CELLS}

Many of the studies on nucleosome assembly and its regulation cited above have been carried out in budding yeast. While many of the RC nucleosome assembly components are conserved from yeast to mammalian cells, nucleosome assembly and its regulation have their own distinct characteristics in mammalian cells. First, in mammalian cells, there are two sequence homologs of Asf1, Asf1a and Asf1b. Like Asf1 in yeast, both Asf1a and Asf1b regulate replication-dependent and replication-independent nucleosome assembly; however, Asf1a and Asf1b appear to have distinct functions. For example, Asf1a, but not Asf1b, interacts with HIRA, a $\mathrm{H} 3-\mathrm{H} 4$ chaperone involved in replication-independent nucleosome assembly (Tagami et al., 2004). Second, to date, there is no clear mammalian homolog of the yeast Rtt106; however, recent studies suggest that the H3.3-H4 histone chaperone DAAX and FACT complex contain regions similar to the yeast Rtt106 (VanDemark et al., 2006; Li et al., 2008; Drane et al., 2010). Third, while H3K56Ac, catalyzed by CBP/P300 and/or Gcn5 (Das et al., 2009; Tjeertes et al., 2009), has been shown to have similar functions in mammalian cells as yeast (Das et al., 2009) (Yuan et al., 2009), it is still unclear as to how this modification regulates nucleosome assembly in mammalian cells, as its abundance is relatively low compared to yeast cells (Jasencakova et al., 2010). Fourth, there are two major forms of $\mathrm{H} 3$ in mammalian cells, canonical H3 (H3.1, H3.2) and histone H3 variant $\mathrm{H} 3.3$, which are deposited by distinct histone chaperones (Ahmad and Henikoff, 2002). Finally, while modifications on newly-synthesized $\mathrm{H} 3-\mathrm{H} 4$ have been extensively profiled in mammalian cells (Loyola et al., 2006; Jasencakova et al., 2010), the functions of these modifications have not been well studied. Therefore, it would be interesting to determine how modifications on newly-synthesized $\mathrm{H} 3-\mathrm{H} 4$ regulate nucleosome assembly pathways in mammalian cells and how this regulation coordinates with concurrent DNA replication.

In summary, over the past few years, we have gained great insight into the regulation of nucleosome assembly through histone modifications and control of histone-histone chaperone interactions. However, there are still many unanswered questions. How does the chromatin modifying machinery aid in the regulation of nucleosome assembly and what regulates these interactions? How does the Asf1- $\mathrm{H} 3-\mathrm{H} 4$ complex pass histone dimers to CAF-1 and/or Rtt106 for deposition? How are parental nucleosomes transferred behind the DNA replication fork? What contributes to histone chaperone specificity for particular $\mathrm{H} 3$ variants? Do modifications on $\mathrm{H} 2 \mathrm{~A}-\mathrm{H} 2 \mathrm{~B}$ facilitate their deposition at the replication fork?

\section{ACKNOWLEDGEMENTS}

R.J.B. is supported by the Mayo Clinic Sydney Luckman Family Predoctoral Fellowship. Z.Z. is a scholar of the Leukemia and Lymphoma Society. Work in the laboratory of Z.Z. is supported by grants from the National Institutes of Health.

\section{REFERENCES}

Ahmad, K., and Henikoff, S. (2002). Histone H3 variants specify modes of chromatin assembly. Proc Natl Acad Sci U S A 99, 16477-16484.

Ai, X., and Parthun, M.R. (2004). The nuclear Hat1p/Hat2p complex: a molecular link between type $B$ histone acetyltransferases and chromatin assembly. Mol Cell 14, 195-205.

Burgess, R.J., Zhou, H., Han, J., and Zhang, Z. (2010). A role for Gcn5 in replication-coupled nucleosome assembly. Mol Cell 37, 469-480.

Carrozza, M.J., Utley, R.T., Workman, J.L., and Côté, J. (2003). The diverse functions of histone acetyltransferase complexes. Trends Genet 19, 321-329.

Chen, C.C., Carson, J.J., Feser, J., Tamburini, B., Zabaronick, S., Linger, J., and Tyler, J.K. (2008). Acetylated lysine 56 on histone $\mathrm{H} 3$ drives chromatin assembly after repair and signals for the completion of repair. Cell 134, 231-243.

Chodaparambil, J.V., Edayathumangalam, R.S., Bao, Y., Park, Y.J., and Luger, K. (2006). Nucleosome structure and function. Ernst Schering Res Found Workshop 57, 29-46.

Das, C., Lucia, M.S., Hansen, K.C., and Tyler, J.K. (2009). CBP/p300mediated acetylation of histone $\mathrm{H} 3$ on lysine 56. Nature 459, 113-117.

Drane, P., Ouararhni, K., Depaux, A., Shuaib, M., and Hamiche, A. (2010). The death-associated protein DAXX is a novel histone chaperone involved in the replication-independent deposition of H3.3. Genes Dev 24, 1253-1265.

Driscoll, R., Hudson, A., and Jackson, S.P. (2007). Yeast Rtt109 promotes genome stability by acetylating histone $\mathrm{H} 3$ on lysine 56 . Science 315, 649-652.

English, C.M., Adkins, M.W., Carson, J.J., Churchill, M.E., and Tyler, J.K. (2006). Structural basis for the histone chaperone activity of Asf1. Cell 127, 495-508.

Falbo, K.B., and Shen, X. (2006). Chromatin remodeling in DNA replication. J Cell Biochem 97, 684-689.

Fillingham, J., Kainth, P., Lambert, J.P., van Bakel, H., Tsui, K., PeñaCastillo, L., Nislow, C., Figeys, D., Hughes, T.R., Greenblatt, J., et al. (2009). Two-color cell array screen reveals interdependent roles 
for histone chaperones and a chromatin boundary regulator in histone gene repression. Mol Cell 35, 340-351.

Goldberg, A.D., Allis, C.D., and Bernstein, E. (2007). Epigenetics: a landscape takes shape. Cell 128, 635-638.

Groth, A., Corpet, A., Cook, A.J., Roche, D., Bartek, J., Lukas, J., and Almouzni, G. (2007). Regulation of replication fork progression through histone supply and demand. Science 318, 1928-1931.

Han, J., Zhou, H., Horazdovsky, B., Zhang, K., Xu, R.M., and Zhang, Z. (2007). Rtt109 acetylates histone H3 lysine 56 and functions in DNA replication. Science 315, 653-655.

Huang, S., Zhou, H., Katzmann, D., Hochstrasser, M., Atanasova, E., and Zhang, Z. (2005). Rtt106p is a histone chaperone involved in heterochromatin-mediated silencing. Proc Natl Acad Sci U S A 102, 13410-13415.

Huang, S., Zhou, H., Tarara, J., and Zhang, Z. (2007). A novel role for histone chaperones CAF-1 and Rtt106p in heterochromatin silencing. EMBO J 26, 2274-2283.

Imbeault, D., Gamar, L., Rufiange, A., Paquet, E., and Nourani, A. (2008). The Rtt106 histone chaperone is functionally linked to transcription elongation and is involved in the regulation of spurious transcription from cryptic promoters in yeast. J Biol Chem 283, 27350-27354.

Jackson, V., Shires, A., Tanphaichitr, N., and Chalkley, R. (1976). Modifications to histones immediately after synthesis. J Mol Biol 104, 471-483.

Jasencakova, Z., Scharf, A.N., Ask, K., Corpet, A., Imhof, A., Almouzni, G., and Groth, A. (2010). Replication stress interferes with histone recycling and predeposition marking of new histones. Mol Cell 37, 736-743.

Jenuwein, T., and Allis, C.D. (2001). Translating the histone code. Science 293, 1074-1080.

Kaufman, P.D., Kobayashi, R., and Stillman, B. (1997). Ultraviolet radiation sensitivity and reduction of telomeric silencing in Saccharomyces cerevisiae cells lacking chromatin assembly factor-I. Genes Dev 11, 345-357.

Kornberg, R.D., and Lorch, Y. (1999). Twenty-five years of the nucleosome, fundamental particle of the eukaryote chromosome. Cell 98, 285-294.

Le, S., Davis, C., Konopka, J.B., and Sternglanz, R. (1997). Two new S-phase-specific genes from Saccharomyces cerevisiae. Yeast 13, 1029-1042.

Li, Q., Fazly, A.M., Zhou, H., Huang, S., Zhang, Z., Stillman, B., and Pearson, C.E. (2009). The elongator complex interacts with PCNA and modulates transcriptional silencing and sensitivity to DNA damage agents. PLoS Genet 5, e1000684.

Li, Q., Zhou, H., Wurtele, H., Davies, B., Horazdovsky, B., Verreault, A., and Zhang, Z. (2008). Acetylation of histone H3 lysine 56 regulates replication-coupled nucleosome assembly. Cell 134, 244-255.

Linger, J., and Tyler, J.K. (2005). The yeast histone chaperone chromatin assembly factor 1 protects against double-strand DNAdamaging agents. Genetics 171, 1513-1522.

Loyola, A., Bonaldi, T., Roche, D., Imhof, A., and Almouzni, G. (2006). PTMs on $\mathrm{H} 3$ variants before chromatin assembly potentiate their final epigenetic state. Mol Cell 24, 309-316.

Luger, K., Mäder, A.W., Richmond, R.K., Sargent, D.F., and Richmond, T.J. (1997). Crystal structure of the nucleosome core particle at $2.8 \mathrm{~A}$ resolution. Nature $389,251-260$.
Ma, X.J., Wu, J., Altheim, B.A., Schultz, M.C., and Grunstein, M. (1998). Deposition-related sites K5/K12 in histone H4 are not required for nucleosome deposition in yeast. Proc Natl Acad Sci U S A 95, 6693-6698.

Marmorstein, R. (2004). Structural and chemical basis of histone acetylation. Novartis Found Symp 259, 78-98; discussion 98-101, 163-109.

Marmorstein, R., and Roth, S.Y. (2001). Histone acetyltransferases: function, structure, and catalysis. Curr Opin Genet Dev 11, 155-161.

Masumoto, H., Hawke, D., Kobayashi, R., and Verreault, A. (2005). A role for cell-cycle-regulated histone $\mathrm{H} 3$ lysine 56 acetylation in the DNA damage response. Nature 436, 294-298.

Mello, J.A., Silljé, H.H., Roche, D.M., Kirschner, D.B., Nigg, E.A., and Almouzni, G. (2002). Human Asf1 and CAF-1 interact and synergize in a repair-coupled nucleosome assembly pathway. EMBO Rep 3, 329-334.

Ransom, M., Dennehey, B.K., and Tyler, J.K. (2010). Chaperoning histones during DNA replication and repair. Cell 140, 183-195.

Recht, J., Tsubota, T., Tanny, J.C., Diaz, R.L., Berger, J.M., Zhang, X., Garcia, B.A., Shabanowitz, J., Burlingame, A.L., Hunt, D.F., et al. (2006). Histone chaperone Asf1 is required for histone H3 lysine 56 acetylation, a modification associated with $S$ phase in mitosis and meiosis. Proc Natl Acad Sci U S A 103, 6988-6993.

Rocha, W., and Verreault, A. (2008). Clothing up DNA for all seasons: Histone chaperones and nucleosome assembly pathways. FEBS Lett 582, 1938-1949.

Roth, S.Y., Denu, J.M., and Allis, C.D. (2001). Histone acetyltransferases. Annu Rev Biochem 70, 81-120.

Ruiz-Carrillo, A., Wangh, L.J., and Allfrey, V.G. (1975). Processing of newly synthesized histone molecules. Science 190, 117-128.

Scholes, D.T., Banerjee, M., Bowen, B., and Curcio, M.J. (2001). Multiple regulators of Ty1 transposition in Saccharomyces cerevisiae have conserved roles in genome maintenance. Genetics 159, 1449-1465.

Shahbazian, M.D., and Grunstein, M. (2007). Functions of sitespecific histone acetylation and deacetylation. Annu Rev Biochem 76, 75-100.

Shibahara, K., and Stillman, B. (1999). Replication-dependent marking of DNA by PCNA facilitates CAF-1-coupled inheritance of chromatin. Cell 96, 575-585.

Sobel, R.E., Cook, R.G., Perry, C.A., Annunziato, A.T., and Allis, C.D. (1995). Conservation of deposition-related acetylation sites in newly synthesized histones $\mathrm{H} 3$ and H4. Proc Natl Acad Sci U S A 92, 1237-1241.

Stillman, B. (1986). Chromatin assembly during SV40 DNA replication in vitro. Cell 45, 555-565.

Strahl, B.D., and Allis, C.D. (2000). The language of covalent histone modifications. Nature 403, 41-45.

Tagami, H., Ray-Gallet, D., Almouzni, G., and Nakatani, Y. (2004). Histone H3.1 and H3.3 complexes mediate nucleosome assembly pathways dependent or independent of DNA synthesis. Cell 116, 51-61.

Tjeertes, J.V., Miller, K.M., and Jackson, S.P. (2009). Screen for DNAdamage-responsive histone modifications identifies H3K9Ac and H3K56Ac in human cells. EMBO J 28, 1878-1889.

Turner, B.M. (2000). Histone acetylation and an epigenetic code. Bioessays 22, 836-845. 
Tyler, J.K. (2002). Chromatin assembly. Cooperation between histone chaperones and ATP-dependent nucleosome remodeling machines. Eur J Biochem 269, 2268-2274.

Tyler, J.K., Adams, C.R., Chen, S.R., Kobayashi, R., Kamakaka, R.T., and Kadonaga, J.T. (1999). The RCAF complex mediates chromatin assembly during DNA replication and repair. Nature 402, 555-560.

Tyler, J.K., Collins, K.A., Prasad-Sinha, J., Amiott, E., Bulger, M., Harte, P.J., Kobayashi, R., and Kadonaga, J.T. (2001). Interaction between the Drosophila CAF-1 and ASF1 chromatin assembly factors. Mol Cell Biol 21, 6574-6584.

VanDemark, A.P., Blanksma, M., Ferris, E., Heroux, A., Hill, C.P., and Formosa, T. (2006). The structure of the yFACT Pob3-M domain, its interaction with the DNA replication factor RPA, and a potential role in nucleosome deposition. Mol Cell 22, 363-374.
Verreault, A., Kaufman, P.D., Kobayashi, R., and Stillman, B. (1996). Nucleosome assembly by a complex of CAF-1 and acetylated histones H3/H4. Cell 87, 95-104.

Vidanes, G.M., Bonilla, C.Y., and Toczyski, D.P. (2005). Complicated tails: histone modifications and the DNA damage response. Cell 121, 973-976.

Wu, J., and Grunstein, M. (2000). 25 years after the nucleosome model: chromatin modifications. Trends Biochem Sci 25, 619-623.

Yuan, J., Pu, M., Zhang, Z., and Lou, Z. (2009). Histone H3-K56 acetylation is important for genomic stability in mammals. Cell Cycle 8, 1747-1753.

Zhou, H., Madden, B.J., Muddiman, D.C., and Zhang, Z. (2006). Chromatin assembly factor 1 interacts with histone $\mathrm{H} 3$ methylated at lysine 79 in the processes of epigenetic silencing and DNA repair. Biochemistry 45, 2852-2861. 